\section{THU0245 EARLY INFLAMMATORY ARTHRITIS CLINICS. DO WE NEED NEW DIAGNOSTIC CRITERIA?}

G Salvador, R Sanmartí, JD Cañete, A Gómez, JR Rodriguez, D Cerdá, J Muñoz-Gómez. Rheumatology, Hospital Clinic, Barcelona, Spain

10.1136/annrheumdis-2001.789

Background The usefulness of the diagnostic criteria for RA (ARA 1987) and spondyloarthropathy (European Group for Spondyloarthropaties 1993) in early inflammatory arthritis remains controversial.

Objectives To analyse the prevalence of inflammatory arthritis according to different diagnostic cathegories, in an early arthritis clinic.

Methods We recruited during one year period (Jan/00-Jan/01) 62 patients $(38 \mathrm{~F}, 24 \mathrm{M})$ mean age 48 ys (range $17-79$ ys), and mean disease duration of $3,1 \mathrm{~m}$ (range 2 wks- $12 \mathrm{~m}$ ). All patients had objective sinovitis in one or more joints with a disease duration between 2 weeks and one year. Septic arthritis and crystal deposition disease have been excluded. Poliarthritis was seen in 37 patients, oligoarthritis in 18 and monoarthritis in 7 . At the time of inclusion 20 out of 62 patients fulfilled ARA (1987) criteria for RA (mean disease duration $4 \mathrm{~m}$ ), 4 patients fulfilled European Group for Spondyloarthopathies criteria (mean disease duration $2 \mathrm{~m}$ ), 2 patients had psoriatic arthritis (mean disease duration $5 \mathrm{~m}$ ), 9 had self-limited arthritis (mean disease duration 3 weeks), in 4 of them an acute infection by PVB19 have been found. In 25 patients arthritis was considered unclassified (mean time disease duration $3 \mathrm{~m}$ ). After a mean time follow-up of 6 months ( $3 \mathrm{w}-1$ yr) 14 out of 20 patients of RA group still fulfilled RA criteria (70\%), the other six fulfilled criteria for different diseases (2 Sjögren sindrome, 1 RS3PE, 1 Polymyalgia, 1 self-limited arthritis and 1 mixed connective tissue disease. Among unclassified arthritis group, 5/25 fulfilled RA criteria and 6/25 patients had oligoarthritis with HLA-B27+ which could suggest a probably spondyloarthropathy despite they didn?t fulfil European Group criteria. Other 3/25 patients developed: 1 Sjögren syndrome, 1 self-limited arthritis and 1 olygoarthritis ANA+ with Hashimoto?s thyroyditis.

Results

Conclusion Present diagnostic criteria for RA, have limited sensitivity and specificity to correctly classify early inflammatory arthritis. Some patients with unclassified arthritis probably have a spondyloarthropathy although they don?t fulfilled the European Group criteria. The development of new diagnostic criteria in patients with early arthritis is necessary.

\section{THU0246 PREVALENCE OF SPECIFIC COMORBID CONDITIONS IN A NATION-WIDE COHORT OF RHEUMATOID ARTHRITIS PATIENTS}

${ }^{1} \mathrm{~L}$ Carmona, ${ }^{2} \mathrm{~A}$ Balsa, ${ }^{3} \mathrm{I}$ González, ${ }^{4} \mathrm{MA}$ Belmonte, ${ }^{5} \mathrm{X}$ Tena, ${ }^{6} \mathrm{R}$ Sanmarti, ${ }^{1}$ Grupo Emecar. ${ }^{1}$ Proyectos, Sociedad Española de Reumatología; ${ }^{2}$ Rheumatology, $\mathrm{H}$ La Paz; ${ }^{3}$ Rheumatology, H La Princesa, Madrid; ${ }^{4}$ Rheumatology, H General, Castellón, Spain; ${ }^{5}$ Rheumatology, H Germans Trias I Pujol, Barcelona; ${ }^{6}$ Rheumatology, $H$ Clinic

\subsection{6/annrheumdis-2001.790}

Background There is compelling evidence that rheumatoid arthritis (RA) may have different expressions and outcomes between populations. Knowing the expression of RA in a determined population should make easier to plan allocation estrategies for health resources and to help predetermine the sample size of future studies.
Objectives To assess the expression of RA in an unselected cohort of patients from various parts of the country. To estimate the prevalence of specific RA features and of comorbid conditions in RA patients from a nation-wide sample.

Methods 780 patients fulfilling the ACR criteria for the classification of RA, and in any stage of the disease, were randomly selected from the registries of 34 participating centres. A baseline visit by trained rheumatologists permitted the investigation of the present expression of the disease and of comorbidities. All comorbid conditions and extraarticular manifestations of RA had protocolised definitions.

Results RA in our country is more frequent in women $(72.0 \%$ [68.9-75.2]), being the mean age at the onset of the disease 41 years \pm 13 (SD). A moderate proportion of patients, $15.4 \%$ [12.8-17.9], fulfilled remission criteria at present. In order of frequency, the estimated prevalence of extra-articular manifestations was: Sjögren's syndrome 16.2\% [13.6-18.7], nodules 16.0\% [13.4-18.6], atlanto-axial subluxation 11.5\% [9.2-13.8], interstitial lung disease 3.2\% [2.0-4.5], eye involvement $2.3 \%$ [1.3-3.4], cardiac and/or pleural effussions $1.6 \%$ [0.7-2.4]. Vasculitis, amyloid, and Felty's syndrome prevalences are below $1 \%$.

The most common comorbidities were, in order of frequency: hypertension 29.7\% [26.5-33.0], cytopenia 27.2\% [24.1-30.4], hypercholesterolemia $24.1 \%$ [21.0-27.1], osteoporosis $21.5 \%$ [18.5-24.4], and depression 10.9\% [8.7-13.1]

Conclusion The preliminary results of this cohort, give us a reliable "picture" of RA in our country. The prevalence of some of the comorbid conditions is higher than expected, despite restrictive definitions. The estimates obtained will be further studied.

This study was sponsored by Aventis, España.

\section{THU0247 THE EPIDEMIOLOGY OF NECK AND LOW BACK PAIN IN THE URBAN POPULATION OF ANTALYA, TURKEY. A PRELIMINARY REPORT}

E Gilgil, C Kacar, B Butun, I Tekeoglu, C Yildirim, G Sumbuloglu, V Arikan, S Urhan, U Dundar, MC Oksuz, T Tuncer. Department of Physical Medicine and Rehabilitation, Akdeniz University Faculty of Medicine, Antalya, Turkey

\subsection{6/annrheumdis-2001.791}

Background Studies elsewhere suggest a lifetime rate of low back pain of about $50-80 \%$ and neck pain of about $12 \%$ in women and $9 \%$ in men.

Objectives To identify the lifetime rate of neck pain and prevalence of low back pain in the urban population of Antalya, Turkey.

Methods This survey study forms a part of larger cross-sectional study still being conducted in the urban area of Antalya (508.840 population according to 1997 national census). So far, 1521 subjects aged 16 or over were interviewed at home visits by 10 trained physicians and 20 accompanying medical students. The subjects were chosen by random cluster sampling from the registries of local offices of the Ministry of Health. Individuals were requested to respond to the questions whether they had at least one episode of neck pain and/or low back pain (both inflammatory and noninflammatory). Additional questions regarding the occurrence of low back pain at present, and in the previous year; loss of work-days in the previous year, the relation of occupation with the symptoms, and whether and how they searched for a solution for the relief of their low back pain were asked to the subjects.

Results We found a lifetime rate of neck pain of $351(46,4 \%)$ of women and $252(33,8 \%)$ of men (totally $611(40,3 \%)$ individuals) 
and low back pain of $430(57 \%)$ of women and $303(\% 40,8)$ of men (totally $739(48,9 \%)$ individuals). The point prevalence of low back pain was calculated as 39,9\%(324 individuals), of these 210 were women and 110 were men. The point prevalence was $27,3 \%(\mathrm{n}=21)$ in men aged over 50 and $34,3 \%(\mathrm{n}=93)$ in men aged 50 or under, while it was $57,6 \%(\mathrm{n}=49)$ in women aged over 50 and 42,5\%(n=161) in women aged 50 or under. Conclusion The lifetime rate of neck and low back pain were both higher in women. The increasing age seems to be associated with the point prevalence of low back pain in women contrary to men.

This study is supported by the Akdeniz University Research Fund.

\section{THU0248 FAMILIAL CLUSTERING OF SYSTEMIC LUPUS ERYTHEMATOSUS WITH OTHER AUTOIMMUNE DISEASES}

${ }^{1} R$ Priori, ${ }^{1} F$ Conti, ${ }^{1} E$ Cassarà, ${ }^{2} E$ Medda, ${ }^{2} M A$ Stazi, ${ }^{3} R$ Gerli, ${ }^{4}$ A Manfredi, ${ }^{5} F$ Franceschini, ${ }^{6} \mathrm{MG}$ Danieli, ${ }^{1} \mathrm{G}$ Valesini. ${ }^{1}$ Rheumatology, "La Sapienza" University; ${ }^{2}$ Istituto Superiore Di Sanità, ISS, Roma; ${ }^{3}$ Rheumatic Diseases, University, Perugia; ${ }^{4}$ URIC, H-S. Raffaele, Milano; ${ }^{5}$ Clinical Immunology, University, Brescia; ${ }^{6}$ Internal Medicine, University, Ancona, Italy

\subsection{6/annrheumdis-2001.792}

Background Previous studies have shown the phenomenon of clustering of autoimmune diseases (AD) in families of patients with Systemic Lupus Erythematosus (SLE).

Objectives The purpose of this study was to evaluate the incidence, prevalence and risk to have an $\mathrm{AD}$ in first degree relatives (FDR) of patients with SLE and to investigate if a history of AD in FDR is a risk factor for SLE.

Methods A multicentre case-control study was carried out in Italy. Cases and controls were randomly selected and interviewed using a detailed questionnaire. We conducted stratified analyses according to sex of relatives and/or proband to evaluate whether the relation between SLE in FDR and AD differs by sex. We used the extended generalised estimating equations (EGEE) to measure familial aggregation. The strenght of the association between family history of AD and SLE was measured as an odds ratio (OR). The adjusted OR was obtained by unconditional logistic regression model and the potentially confounding covariates were family size, age and sex of proband.

Results The prevalence of AD among SLE and control FDR was respectively $6.1 \%$ and $1.5 \%(\mathrm{p}<0.01)$. The incidence rate of $\mathrm{AD}$ and control FDR was $1.35 \times 1000$ py and $0.32 \times 1000$ py respectively. The relatives of SLE cases have 4 time probability of having AD compare to relatives of control (RR 4.26; 95\% CI 2.25- 8.03). Using EGEE the adjusted OR taking into account the non-independence of family members was 4.77 (95 CI 2.0711). A risk of 8.57 (95\% CI 3.18-23.1) was detected if the proband is female and $1.40(95 \%$ CI $0.22-8,78)$ if proband is male. The risk of AD is significantly greater for female with a female FDR with SLE (OR 10.36; 95\% CI 2.90- 36.9). No AD was found among SLE spouses, while one case was found among spouses of controls.

Conclusion This case-control study defines the risk to have an autoimmune disorder for SLE first degree relatives. A family history for $\mathrm{AD}$ represents a risk factor for SLE.
THU0249 FAMILY HISTORY OF AUTOIMMUNE DISEASES AS A RISK FACTOR FOR SJÖGREN'S SYNDROME: A CASECONTROL STUDY

${ }^{1} \mathrm{R}$ Priori, ${ }^{1} \mathrm{~F}$ Conti, ${ }^{1} \mathrm{E}$ Cassarà, ${ }^{2} \mathrm{E}$ Medda, ${ }^{2} \mathrm{MA}$ Stazi, ${ }^{3} \mathrm{M}$ Cesarotti, ${ }^{4} \mathrm{P}$ Rovere, ${ }^{5} \mathrm{C}$ Antonioli, ${ }^{6} \mathrm{MG}$ Danieli, ${ }^{7} \mathrm{M}$ Pietrogrande, 'G Valesini. 'Rheumatology, "La Sapienza" University; ${ }^{2}$ Istituto Superiore Di Sanità, ISS, Rome; ${ }^{3}$ Rheumatic Diseases, University, Perugia; ${ }^{4} U R I C, H$ S. Raffaele, Milano; ${ }^{5}$ Clinical Immunology, University, Brescia; ${ }^{6}$ Internal Medicine, University, Ancona, Italy; ${ }^{7} \mathrm{H}-\mathrm{S}$. Paolo, University

10.1136/annrheumdis-2001.793

Background As far as we know, no epidemiological study is available regarding risk factors for Sjögren's syndrome (SS).

Objectives A multicentre case-control study was carried out in Italy to evaluate the relationship between SS and a history of autoimmune diseases (AD) in first degree relatives (FDR).

Methods Cases and controls were randomly selected and interviewed by trained medical doctors using a detailed questionnaire. The strenght of the association between family history of $\mathrm{AD}$ and SS was measured as an odds ratio (OR). The adjusted OR was obtained by unconditional logistic regression model and the potentially confounding covariates were family size, age and sex of proband.

Results A total of 143 eligible SS cases (EEC criteria,1994) and 140 healthy controls were identified. 27/143 (18.8\%) SS and 7/ $140(5 \%)$ controls had one FDR with $\mathrm{AD}, 7 / 143$ (4.9\%) and 1/ $140(0.7 \%)$ had two or more FDR with AD. Considering the proband with one FDR with AD (therefore belonging to a multiplex family) in comparison to probands without any FDR affected (belonging to a simplex family), the adjusted OR was 6.19 (95\% CI 2.3-16.7); the risk is higher if the relatives are two or more: OR 19.2 (95\% CI 1.69-216).

Conclusion This study indicates that a family history of autoimmune disorders is a risk factor for SS. Probands from multiplex families have an higher risk to have SS compared to simplex families. The risk increases with the number of FDR with AD.

\section{THU0250 AUTOIMMUNE DISORDERS IN ICELANDIC MULTICASE SLE FAMILIES}

G Gröndal, K Steinsson. Center for Rheumatology Research and Department of Rheumatology, Landspitalinn, University Hospital, Reykjavik, Iceland

\subsection{6/annrheumdis-2001.794}

\section{Background}

Objectives To evaluate the frequency of autoimmune disorders in Icelandic multicase SLE families.

Methods The study group consisted of 38 SLE patients, 127 first-degree relatives, 37 s-degree relatives and 33 nonconsangineous relatives from multicase SLE families. Pedigree members were evaluated through interviews and physical examination, medical records were reviewed. A standardised protocol for SLE and other autoimmune disorders was used. Serum samples were obtained and autoantibodies measured.

Results Among the SLE patients, 16/38 (42\%) had other autoimmune disorder (s) (11 Sjogrens syndrome/sicca symptoms, 3 interstitial cystitis, 3 psoriasis, 3 pernicious anaemia, 3 thyroid disease, 2 myositis). Twenty six/164 (16\%) first- and seconddegree relatives had various autoimmune disorder (s) (14 Sjogrens syndrome/sicca symptoms, 8 thyroid disease, 6 RA, 3 psoriasis, 3 alopecia, 3 iritis, 1 pernicious anaemia, 1 PBC, 1 MS, 1 DM, 1 AS, 1 reactive athritis, 1 discoid LE). Additionally, 21/ $164(13 \%)$ relatives had autoantibodies. Therefore either 